\title{
Understanding the impact of antibiotic perturbation on the human microbiome
}

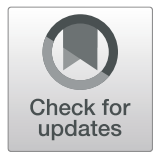

Drew J. Schwartz ${ }^{1,2^{*}+}$ (D), Amy E. Langdon $2,3+$ and Gautam Dantas $2,3,4,5^{*}$

\begin{abstract}
The human gut microbiome is a dynamic collection of bacteria, archaea, fungi, and viruses that performs essential functions for immune development, pathogen colonization resistance, and food metabolism. Perturbation of the gut microbiome's ecological balance, commonly by antibiotics, can cause and exacerbate diseases. To predict and successfully rescue such perturbations, first, we must understand the underlying taxonomic and functional dynamics of the microbiome as it changes throughout infancy, childhood, and adulthood. We offer an overview of the healthy gut bacterial architecture over these life stages and comment on vulnerability to short and long courses of antibiotics. Second, the resilience of the microbiome after antibiotic perturbation depends on key characteristics, such as the nature, timing, duration, and spectrum of a course of antibiotics, as well as microbiome modulatory factors such as age, travel, underlying illness, antibiotic resistance pattern, and diet. In this review, we discuss acute and chronic antibiotic perturbations to the microbiome and resistome in the context of microbiome stability and dynamics. We specifically discuss key taxonomic and resistance gene changes that accompany antibiotic treatment of neonates, children, and adults. Restoration of a healthy gut microbial ecosystem after routine antibiotics will require rationally managed exposure to specific antibiotics and microbes. To that end, we review the use of fecal microbiota transplantation and probiotics to direct recolonization of the gut ecosystem. We conclude with our perspectives on how best to assess, predict, and aid recovery of the microbiome after antibiotic perturbation.
\end{abstract}

Keywords: Gut microbiome, Resistome, Antibiotics, Perturbation, Resilience, Dynamics, Recolonization

\section{Introduction}

The human gut microbiome consists of bacteria, viruses, and fungi ideally living symbiotically with their human host, though this review will focus exclusively on bacterial residents within the gut microbiome [1]. Individual species and collective bacterial functions within the gut microbiome confer many benefits throughout life including metabolizing dietary

\footnotetext{
* Correspondence: Schwartzd@wustl.edu; Dantas@wustl.edu

${ }^{\dagger}$ D. J. Schwartz and A. E. Langdon contributed equally to this work. 'Department of Pediatrics, Division of Infectious Diseases, Washington University School of Medicine in St. Louis, St. Louis, MO 63110, USA

${ }^{2}$ The Edison Family Center for Genome Sciences \& Systems Biology,

Washington University School of Medicine in St. Louis, St. Louis, MO 63110, USA

Full list of author information is available at the end of the article
}

contributions, educating the immune system, defending against pathogens, and contributing to overall health and optimal growth [2-6]. The gut microbiome is affected by and influences pathologies including inflammatory bowel disease (IBD), allergies, asthma, and neurobehavioral disorders [4, 5, 7, 8]. Another key feature of the microbiome is the quantity, identity, and function of antibiotic resistance genes (ARGs), collectively called the resistome. ARGs transmit between species within the gut microbiome including potential pathogens. Therefore, understanding how the resistome changes in parallel with the microbiome is vitally important $[9,10]$. Accordingly, numerous avenues of research are being pursued to

(C) The Author(s). 2020, corrected publication 2021. Open Access This article is licensed under a Creative Commons Attribution 4.0 International License, which permits use, sharing, adaptation, distribution and reproduction in any medium or format, as long as you give appropriate credit to the original author(s) and the source, provide a link to the Creative Commons licence, and indicate if changes were made. The images or other third party material in this article are included in the article's Creative Commons licence, unless indicated otherwise in a credit line to the material. If material is not included in the article's Creative Commons licence and your intended use is not permitted by statutory regulation or exceeds the permitted use, you will need to obtain permission directly from the copyright holder. To view a copy of this licence, visit http://creativecommons.org/ licenses/by/4.0/. The Creative Commons Public Domain Dedication waiver (http://creativecommons.org/publicdomain/zero/1. 0/) applies to the data made available in this article, unless otherwise stated in a credit line to the data. 
understand what constitutes healthy and abnormal microbiomes and resistomes.

Current microbiome research is largely concerned with "who is there?" and "what are they doing?" Microbiome taxonomic profiling is achieved by culturedependent molecular or phenotypic typing or cultureindependent sequencing of taxonomically informative marker genes or whole metagenomic shotgun sequencing from microbiome samples, within or between individuals [11-14]. Similarly, features and functions of the microbiome can be assessed by gene-level analysis, metabolomics, and assessment of the abundance of gene pathways for microbial metabolic function [15-20]. These analyses are typically conducted in the context of human development throughout life or in connection with clinical outcomes [21]. Measures of diversity within $(\alpha)$ and between $(\beta)$ samples can be used to compare microbial communities over time and between disease states (extensively reviewed in [22]). Our ability to attribute disease associations to causality is difficult and requires longitudinal, prospective studies, ideally complemented by mechanistic validation in animals [21, 23]. However, important associations between the human microbiome structure and function with diseases and health nevertheless provide meaningful hypotheses and correlations [7, 21, 24, 25].

The most common external perturbations to the microbiome are diet, medications (especially antibiotics), and the environment [26-31]. In this review, we focus on antibiotic perturbation throughout life and associations with other factors including age and maturity of the microbiome, diet and the environment, and the co-morbidities of the individual (Fig. 1). How the microbiome responds to antibiotics is altered by the state of the microbiome at the time of perturbation (diet, species, and functional diversity and redundancy) and the strength of the perturbation (route, spectrum, and duration of antibiotics). After cessation of antibiotics, the prevalence of beneficial or potentially pathogenic and/or antibiotic-resistant (AR) microbes that recolonize the gut microbiome governs the initial and long-term outcomes of antibiotic treatment (Fig. 1). These factors must be considered individually and collectively when correlating changes in the microbiome structure and function to human health and disease.

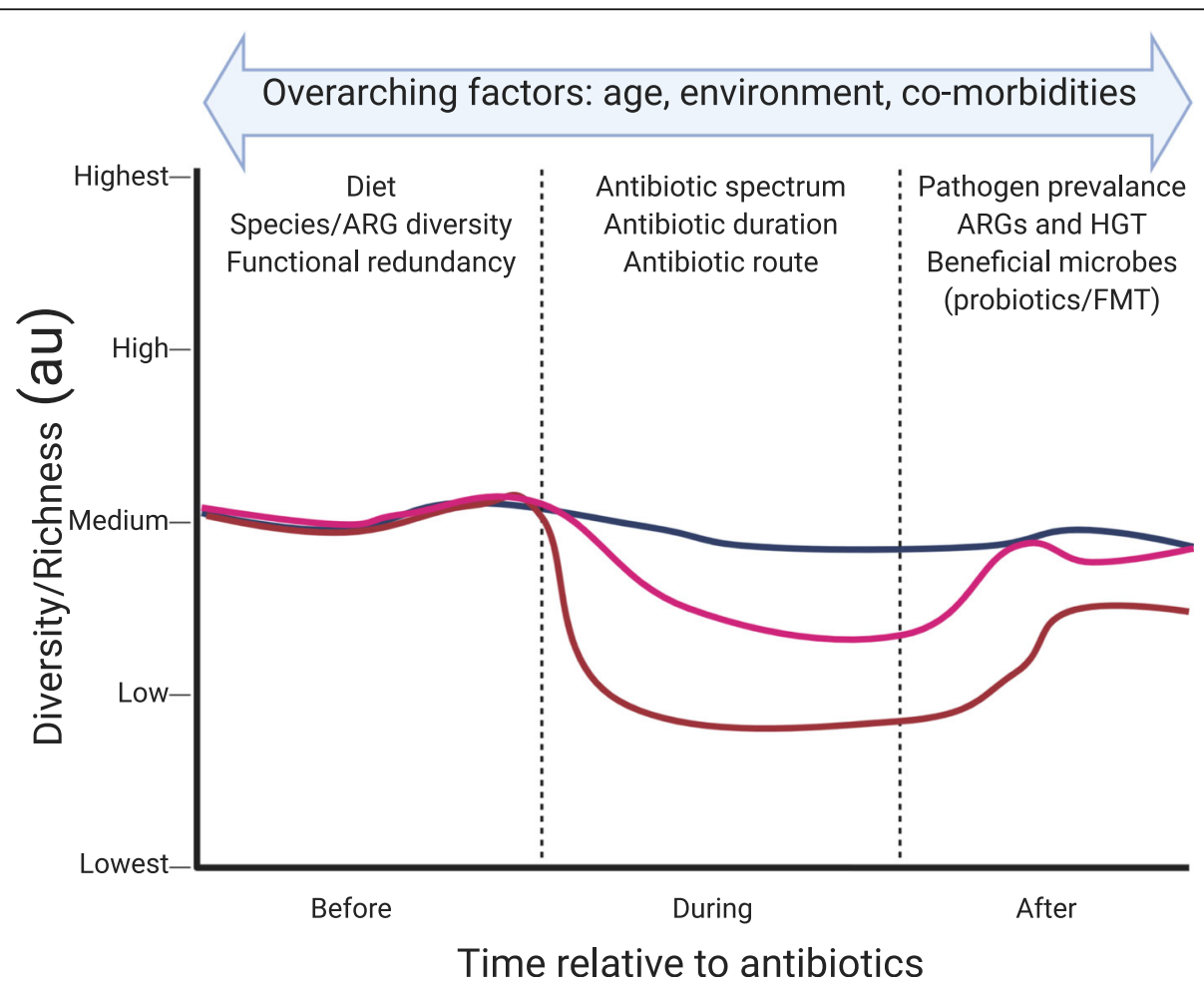

Fig. 1 Antibiotic perturbation to the microbiome needs to be considered in context. Certain factors are important to consider throughout life (overarching factors). Other factors such as diet and the functional and species diversity and redundancy are important to consider when the antibiotic perturbation is applied. The duration, spectrum, and route of antibiotics are vitally important in the context of how the microbiome responds during an intervention. The post-antibiotic environment including availability and colonization of pathogens, frequency of horizontal gene transfer (HGT), MDROs, and beneficial microbes is important to consider the resilience and response after antibiotic cessation. These factors influence the structure and function of the microbiome before, during, and after antibiotics throughout life. Created with BioRender 


\section{Development of the human microbiome}

Immediately after birth, bacteria, viruses, and fungi colonize the sterile human gut with a subsequent rapid accumulation of species and diversity from the mother and the environment [32-34]. Gut microbiome richness and diversity continue to change until 2-3 years of life, after which the microbiome stabilizes with less dramatic monthly changes $[26,35]$. The neonatal and infant periods, however, are defined by dynamic changes in microbial diversity on a weekly, if not daily, timescale. Dramatic strain-level changes and interactions shape the early life microbiome providing essential metabolic and immune regulatory functions [36, 37]. This increase in microbial species and functions matures the gut microbiome increasing both its richness and diversity [26, 38]. Determining geographical and environmental developmental baselines and trajectories allows for understanding the effects of perturbations to normal development $[32,39]$. Preterm birth, residence in the neonatal intensive care unit (NICU), malnutrition, and frequent antibiotics can perturb microbiome development and result in microbiota "age regression" [32, 38, 39]. Microbiota age regression is indicated by the child's chronological age exceeding their age predicted from the constituents of their microbiome [32, 39]. Thus, comparing a child's chronological age with an assessment of microbiota health from the species composition can provide a gross understanding of development and disruptions thereof.

The overall diversity and community architecture of healthy adult microbiomes do not change dramatically in the absence of significant perturbation [26, 40, 41]. However, among healthy adults, the relative abundance of specific taxa can change on a daily or weekly scale [42], but microbial functions are more stable longitudinally [43]. As an important caveat, some of these inferred differences regarding daily changes may be an erroneous

Table 1 Microbiome methods and limitations

Bias introduced during extraction, amplification, sequencing, and bioinformatic processing can alter the relative abundances of species within a sample [44]. Relative abundances can range from 50-fold higher or lower than actual depending on the specific species contribution and protocols used [44]. The complete absence of a species may reflect bias below the limit of detection. Conversely, expansion of specific taxa may reflect progressive and systemic bias enriching for sequencing reads from those taxa [44]. It is therefore important to consider and correct for these biases in any experiment where taxon relative abundance is considered using computational methods [44]. A key step in any metagenomic sequencing experiment is to sequence similar, defined communities of different taxon proportions to understand bias in each protocol. Sequencing-defined communities can lead to computational estimates of protocol bias that can be applied to all samples prior to analysis [44]. Furthermore, extraction and processing introduce contamination depending on its format, and each kit has its own DNA that needs to be evaluated especially when considering a potentially sterile site [45-47]. Thus, sequencing both mock, negative controls of the sequencing kit only and contrived, defined bacterial communities is essential for optimal microbiome sequencing determination. result of sequencing error from various sources and amplification of or depletion of rare and low abundance taxa (Table 1) $[44,48]$. Biases can be both taxa-intrinsic as well as protocol-specific [44]. For example, among 3 different extraction protocols, the relative abundance of Clostridioides difficile was universally lower and Fusobacterium nucleatum higher than the actual abundance of the mock community [44]. Conversely, certain protocols enriched for specific taxa over others in all mock communities. The range of individual abundances among replicates varied as much as 10 -fold within a given extraction and processing protocol [44]. Therefore, a daily change in relative abundance could be a result of systematic bias and should be evaluated as such (Table 1).

Although the dichotomy of stable (e.g., healthy adult) versus dynamic (e.g., developing infant) microbiomes is likely oversimplified, it is important to understand that the impact of an intervention/perturbation depends on the context (Fig. 1). That is, a relatively more stable, healthy, adult microbiome can resist and rebound faster and more completely from the same perturbation that could change the developmental trajectory of a preterm neonate and leave lasting changes (Fig. 1) [32]. Partly, this difference relates to the degree of functional diversity and redundancy in the microbiome with different strains of the same species contributing functions or occupying various distinct and overlapping niches [37, 49].

\section{Acute perturbations to the microbiome and resistome}

\section{Vulnerable infancy}

Large-scale studies of the microbiome have demonstrated that the dynamic first 2 years of life respond most dramatically to antibiotic perturbation [26, 50]. Antibiotic treatment during the first 18 months of life results in greater disruption than subsequent administration, as measured by $\beta$ diversity between consecutive samples [50, 51]. Among the most dynamic periods of microbiome development is the first 6 months of life $[26,28]$. Prior to birth, intrapartum antibiotic administration to mothers significantly affected the microbiome structure of 1-month-old neonates relative to control infants even in the absence of continued antibiotic exposure [52, 53] (Table 2). Further, intrapartum antibiotics lead to persistent enrichment of ARGs in exposed, term neonates relative to non-treated neonates or their mothers at 6 months of life [52]. Importantly, in this study, neither exposed nor the control children were antibiotic-treated after birth. Therefore, the administration of antibiotics during this critical developmental window can lead to short- and intermediate-term negative effects on the microbiome and resistome.

An extreme example of this paradigm of microbiome dynamics is neonates who are born prematurely and 
Table 2 Key findings of summarized work

\begin{tabular}{|c|c|c|c|}
\hline Authors & Population & General findings & Species and ARGs implicated \\
\hline Parnanen et al. 2018 [52] & $\begin{array}{l}\text { Fecal samples of } 16 \text { mother-infant } \\
\text { pairs shotgun metagenomic } \\
\text { sequenced over the first } 6 \text { months } \\
\text { of life }\end{array}$ & $\begin{array}{l}\text { Intrapartum antibiotics increased } \\
\text { fetal ARGs and decreased diversity } \\
\text { at } 1 \text { month }\end{array}$ & $\begin{array}{l}\text { Efflux pumps and other ARGs mapping } \\
\text { to E. coli and Klebsiella spp. enriched in } \\
\text { antibiotic-exposed subjects }\end{array}$ \\
\hline Gibson et al. 2016 [54] & $\begin{array}{l}84 \text { NICU-hospitalized } \\
\text { preterm neonates with stool } \\
\text { samples flanking antibiotic } \\
\text { treatment sequenced }\end{array}$ & $\begin{array}{l}\text { Meropenem, cefotaxime, and } \\
\text { ticarcillin-clavulanate decreased } \\
\text { microbiome diversity whereas } \\
\text { gentamicin and vancomycin } \\
\text { had variable effects }\end{array}$ & $\begin{array}{l}\text { Abundance of E. coli and } S \text {. aureus and } \\
\text { the two-component regulator system, } \\
c p \times R / c p x A \text { predicted gut microbiome } \\
\text { response to vancomycin and gentamicin }\end{array}$ \\
\hline Bokulich et al. 2016 [55] & $\begin{array}{l}43 \text { infants followed over the first } \\
2 \text { years of life }\end{array}$ & $\begin{array}{l}\text { Antibiotics delayed microbiome } \\
\text { maturation with fewer species } \\
\text { and lower diversity that resolved } \\
\text { after } 1 \text { year of life }\end{array}$ & $\begin{array}{l}\text { Relative abundance of Clostridiales } \\
\text { and Ruminococcus decreased from } \\
3 \text { to } 9 \text { months in the antibiotic- } \\
\text { exposed group }\end{array}$ \\
\hline Palleja et al. 2018 [56] & $\begin{array}{l}12 \text { healthy adults treated with } 4 \text { days } \\
\text { of meropenem, gentamicin, and } \\
\text { vancomycin with fecal shotgun } \\
\text { metagenomic sequencing for } \\
6 \text { months after }\end{array}$ & $\begin{array}{l}\text { Gut microbiome diversity } \\
\text { recovered after } 6 \text { months, but } \\
\text { richness did not; no persistent } \\
\text { enrichment of ARGs }\end{array}$ & $\begin{array}{l}\text { Multi-drug efflux pumps most enriched } \\
\text { immediately after treatment; complete } \\
\text { absence at } 6 \text { months of baseline species } \\
\text { belonging to Bifidobacterium, Coprococcus, } \\
\text { and Methanobrevibacter within individuals }\end{array}$ \\
\hline Lloyd-Price et al. 2019 [7] & $\begin{array}{l}\text { Multi-omic analysis of } 132 \\
\text { children and adults with IBD } \\
\text { or controls contributing } 2965 \\
\text { specimens }\end{array}$ & $\begin{array}{l}\text { Increased inter-individual } \\
\text { variation during IBD flare; } \\
\text { multi-omic signatures } \\
\text { differentiate dysbiosis from } \\
\text { baseline }\end{array}$ & $\begin{array}{l}\text { Prevotella copri maintained high } \\
\text { relative abundance in Crohn's disease } \\
\text { patients but fluctuated in its abundance } \\
\text { in controls; dysbiosis marked by decreased } \\
\text { Faecalibacterium prausnitzii and Roseburia } \\
\text { hominis and increased E. coli }\end{array}$ \\
\hline Gasparrini et al. 2019 [32] & $\begin{array}{l}41 \text { NICU-hospitalized preterm } \\
\text { infants variably exposed to } \\
\text { antibiotics and } 17 \text { antibiotic- } \\
\text { naive near-term infants followed } \\
\text { through } 21 \text { months of life }\end{array}$ & $\begin{array}{l}\text { Preterm infant microbiome } \\
\text { exhibited delayed development } \\
\text { with recovery by } 15 \text { months }\end{array}$ & $\begin{array}{l}\text { Persistent MDRO Enterobacteriales } \\
\text { colonization in several infants; model } \\
\text { including Prevotella copri, Eubacterium } \\
\text { rectale, Ruminococcus spp., and ARGs } 96 \% \\
\text { predictive of whether a fecal sample } \\
\text { originated from a preterm, antibiotic- } \\
\text { exposed or near-term antibiotic-naive infant }\end{array}$ \\
\hline Yassour et al. 2016 [28] & $\begin{array}{l}39 \text { Finnish children aged } 2 \text { to } \\
36 \text { months contributing monthly } \\
\text { stool samples }\end{array}$ & $\begin{array}{l}\text { Frequent antibiotic courses } \\
\text { diminished gut microbiome } \\
\text { species and strain diversity } \\
\text { and enriched for ARGs }\end{array}$ & $\begin{array}{l}\text { Antibiotic treatment more drastically } \\
\text { affected the strain-level diversity of } \\
\text { Bacteroides fragilis than Bacteroides } \\
\text { vulgatus; relative abundance of many } \\
\text { ARGs decreased after cessation; others } \\
\text { (CfxA6 beta-lactamase) remained high }\end{array}$ \\
\hline Doan et al. $[57,58]$ & $\begin{array}{l}30 \text { children in Niger randomized } \\
\text { to placebo or bi-annual azithromycin } \\
\text { for } 2 \text { years }\end{array}$ & $\begin{array}{l}\text { No dramatic effect on microbiome } \\
\text { diversity or relative abundance }\end{array}$ & $\begin{array}{l}\text { Decreased relative abundance of } \\
\text { Campylobacter spp.; increased } \\
\text { macrolide resistance overall and in } \\
\text { S. pneumoniae at } 24 \text { months }\end{array}$ \\
\hline Suez et al. 2018 [59] & $\begin{array}{l}21 \text { healthy adults treated with } \\
7 \text { days of ciprofloxacin and } \\
\text { metronidazole then randomized } \\
\text { to probiotics, autologous FMT, } \\
\text { and spontaneous recovery }\end{array}$ & $\begin{array}{l}\text { FMT accelerated and probiotics } \\
\text { inhibited microbiome structural } \\
\text { and functional recovery }\end{array}$ & $\begin{array}{l}\text { Relative abundance of Enterococcus } \\
\text { casseliflavus and Blatia producta } \\
\text { inversely correlated with overall } \\
\text { microbiome richness }\end{array}$ \\
\hline
\end{tabular}

reside in NICUs. Antibiotic treatment of premature infants is both routine and extensive [60-62]. Antibiotic treatment decreases gut microbial diversity and enriches for AR potential pathogens [54]. Antibiotics delivered in the NICU are varied, [63] ranging from relatively shortterm exposure with narrow-spectrum agents such as ampicillin or cefazolin to long-term exposure with broad-spectrum agents such as 3rd-generation cephalosporins and carbapenems [54, 64]. The preterm gut microbiota of NICU-hospitalized neonates is dominated by Escherichia coli, Klebsiella spp., Enterobacter spp., and Enterococcus spp., which are found in the NICU environment, are often multi-drug resistant, and are causes of bacteremia in this population $[54,65,66]$ (Table 2). Antibiotic administration in this environment results in a fundamentally altered and extremely ARGenriched gut microbiome acutely after antibiotics, but the response to each antibiotic differed based on the microbiome composition [54]. The overall microbiome response to gentamicin and vancomycin could be predicted based on the abundance of the species E. coli and Staphylococcus aureus and the ARGs/bacterial response regulator $c p x A / c p x R$ [54]. Additionally, members of the Enterobacteriales harbored hundreds of novel ARGs 
conferring functional resistance to beta-lactams, tetracyclines, and aminoglycosides [54]. Further research investigating the strain-level diversity and functional evolution over time in the NICU and after discharge is needed to identify the covariation and consequences of specific antibiotic therapy in the context of birth history, diet, and environment for each individual's microbiome.

Antibiotic treatment in infancy enriches for AR organisms in the stool as determined by selective culturing and DNA sequencing [32, 54]. To more broadly characterize novel and unknown resistance mechanisms, researchers have utilized functional metagenomics, whereby fecal DNA is cloned into plasmids, introduced into E. coli, and plated on selective media [32, 54, 67]. These functionally validated ARGs present in the original fecal samples encode for proteins, the majority of which had not been previously ascribed resistance mechanisms in curated databases [54, 68]. Greater than $40 \%$ of these ARGs derive from E. coli, Enterobacter spp., and Klebsiella spp., encoding resistance to commonly used antibiotics in the NICU (penicillins and cephalosporins) as well as antibiotics not used in the NICU (tetracyclines and chloramphenicol) [54] (Table 2). A strength of this approach is that it allows the identification of a broad range of ARGs assuming expression in E. coli. Accordingly, these novel ARGs are likely still an underestimate of the total resistome. To date, similar methods have been unsuccessful in gram-positive bacteria. Resistance to antimicrobials that exclusively or preferentially target gram positives such as vancomycin, linezolid, and clindamycin would require other methods to identify. Therefore, frequent antibiotic use during times of microbial change acutely disturbs the microbiome and enriches for potential pathogens and ARGs.

\section{Dynamic childhood}

Childhood is a time of immense microbiome dynamics and environmental changes including dietary shifts and introductions $[6,26,69]$. After vulnerable infancy, the microbiome goes through a developmental stage (3-14 months of age) and a transitional stage (15-30 months) before stabilizing to a near-adult like configuration [26]. Arguably, the most significant microbiome perturbation during early childhood is the transition from breastmilk or formula to solid foods $[26,70]$. The pre-weaning microbiome is dominated by Bifidobacterium spp. and, thereafter, several microbiome states, so-called enterotypes, account for the bulk of gut community structure [26]. Microbial diversity of a breastfed child after weaning increases commensurate with the accumulation and enrichment of strains responsible for dietary metabolism [26, 27, 37]. Transitions between these states accompany chronological aging, likely reflective of differential environmental exposures including antibiotics, infections, and diet $[26,55,69]$.
Antibiotic administration during childhood typically consists of short courses of relatively narrow spectrum agents for respiratory tract and oropharyngeal infections [71]. Intermittent postnatal antibiotic exposure was associated with decreased abundance of Clostridiales and Ruminococcus even though the overall number of species and diversity was similar between exposed and unexposed groups after 1 year of life (Table 2) [55]. However, if antibiotics are frequent or in the context of underlying gastrointestinal disturbances or inadequate diet, their effects can be magnified [55, 72]. Specifically, short courses of antibiotics were shown to exacerbate dysbiosis from Crohn's disease [72]. Thus, antibiotics, diet, and environment acutely impact the developmental trajectory and diversity of the gut microbiome of the developing child.

\section{Stable adulthood}

A child's microbiome achieves an adult-like configuration with less dramatic changes monthly between 2 and 3 years of age [26, 35]. Assuming that the healthy adult microbiome is completely stable is inaccurate as high temporal resolution studies have shown variability of different body sites over time [48]. However, individuals with the greatest Shannon diversity in the gut microbiome had the smallest temporal variability with most taxa stable over long periods of time in the absence of perturbation $[41,48]$. These findings have ushered in one of the central dogmas of the microbiome field: microbiomes with higher diversity are more resilient to perturbation. For instance, researchers treated 12 men with 4 days of a cocktail of broad-spectrum antibiotics (meropenem, gentamicin, and vancomycin) and sequenced their gut microbiome over a 6-month period [56] (Table 2). Immediately after treatment, Klebsiella spp., Enterococcus spp., and E. coli increased in abundance, but by 8 days after treatment, no significant differences were observed in these species, suggesting acute bacterial blooms are short-lived after cessation of antibiotics in this population. Microbial richness and diversity dramatically decreased, but Shannon diversity progressively recovered in the subsequent 6 months. Although at the gross community level, the microbiome was restored, the absolute number of species remained significantly lower from baseline samples [56].

Lumping together all antibiotics is an oversimplification as the antibiotic spectrum clearly impacts the response of the gut microbiome (Fig. 1) [73]. Indeed, computational modeling of the short-term impact of various antibiotics demonstrates that ciprofloxacin, a broad-spectrum fluoroquinolone antibiotic, showed more displacement from microbiome equilibrium than amoxicillin, a narrow spectrum $\beta$-lactam antibiotic [74]. Certain strains of the same species do not recover, 
suggesting potentially lasting consequences. Similarly, oral cefprozil (a $\beta$-lactam antibiotic) altered strain-level dynamics shifting the dominant strain within each individual $[75,76]$. It is likely that some of the individualization of antibiotic effects on strain-level diversification is due to the copy number variation or expression of specific ARGs [76]. Although certain ARGs were significantly increased immediately after antibiotic treatment, reports of lasting ARG changes vary per study likely reflective of the microbiome composition, spectrum, and route of antibiotic administration, comorbidities, and other factors (Fig. 1) [56, 75, 76]. However, if a stable microbiome is challenged simultaneously by changing the environment such as foreign travel to high infectious burden areas or intensive care admission and antibiotic treatment, the acute microbiome effects can be more dramatic [77-79]. These results demonstrate the myriad acute changes to the microbiome and suggest the remarkable plasticity of the stable adult microbiome to routine perturbation.

Although the adult microbiome is relatively stable in healthy adults, the microbiome dynamically changes during acute inflammation of gastrointestinal illnesses such as IBD [7] (Table 2). In one study, researchers characterized samples as dysbiotic by computing Bray-Curtis dissimilarity between all samples to a reference set of non-IBD controls. Any samples that scored above the 90th percentile from the median sample-sample difference from the reference set were termed dysbiotic. Using a multi-omics approach, they identified that $24 \%$ of samples from patients with Crohn's disease were dysbiotic both metagenomically and metabolomically, far above the expected $10 \%$ threshold. Similarly, during dysbiotic shifts, patients had discursions from expected constitution not only of metagenomes and their associated metatranscriptomes and metabolomes, but also in measurements of inflammation [7]. Both concurrent and antibiotic use in the prior 6 months correlated with gut dysbiosis further exacerbating the inter-individual and intra-individual microbiome compositional differences $[72,80]$. Thus, acute, antibiotic perturbations to the microbiome have a greater effect on community structure when the microbiome is dynamically changing due to inflammation.

Another potentially more dynamic time of microbiome structure is in the elderly. Indeed, small studies have shown decreased microbiome diversity in the elderly including decreased Firmicutes and increased Proteobacteria, similar to the neonatal microbiome [81-83]. In contrast to younger adults, the microbiome was more variable in its composition when sampled within 3month intervals [83]. One potential explanation for this finding is the number and diversity of non-antibiotic medications that have been shown to impact the microbiome [31]. More research needs to be conducted on the elderly microbiome in the context of immune senescence, co-morbidities, and infection susceptibility, but the stability of the human microbiome may decrease at either extreme of age.

\section{Long-term effects of antibiotic perturbation depend on microbiome state}

Understanding the long-term effects of specific antibiotics is vital to limiting the negative consequences of AR development and international spread. Despite the overall stable community structure over time, changes in species composition may persist even after short antibiotic perturbations to the stable microbiome $[56,75,84]$. The immediate environment of an antibiotic recipient is an important source of newly introduced microbes [32, 54, $59,66,85,86]$. This simple fact deserves a great deal more attention to ensure reliable, positive long-term outcomes after perturbations to the gut microbiome. Longer-term effects are also highly dependent on stage of life and stability of the microbiome (Fig. 1) [26, 28, 32, 50, 87, 88].

\section{Vulnerable infancy and dynamic childhood}

Frequent antibiotic use in the NICU delays preterm neonatal microbiome maturation initially, but the microbiota recovers to a similar taxonomic composition to antibiotic-naive term controls by 15 months of life [32]. Similar microbiome recovery at 4 years of life has been shown for antibiotic treatment in the first year of life [69]. Thus, even in periods of extreme microbiome turnover, from a gross, structural level, the microbiome is still resilient and converges to a similar architecture with antibiotic-naive infants. Although the overall composition of the microbiome was similar, specific species and ARGs present up to 2 years later distinguished them from healthy controls [32] (Table 2). Specifically, multidrug resistant organism (MDRO) Enterobacteriales ( $E$. coli, Klebsiella spp., and Enterobacter spp.) acquired in the NICU persisted in the gut microbiome up to 1 year after NICU discharge with greater than $99.997 \%$ identity between them. Machine learning algorithms classified fecal samples post-NICU discharge deriving from an antibiotic-naive or frequently exposed infant with $96 \%$ accuracy [32]. Among the most significant features distinguishing these samples were ARGs including class A $\beta$-lactamases as well as functionally validated ARGs to piperacillin and tetracycline and members of the order Clostridiales.

In addition to allowing MDRO potential pathogens to maintain a foothold within the GI tract, frequent antibiotic exposure in this critical microbiome developmental period diminished strain diversity, enriched for ARGs, and conferred a less stable composition [28]. Researchers collected monthly stool samples from 39 children from 2 months to 3 years variant in their antibiotic 
usage [28] (Table 2). Twenty of these children received between 9 and 15 antibiotic courses mainly for otitis media and respiratory infections. They found that antibiotic-naive children harbored increased strain-level diversity of Bacteroides fragilis, a key commensal important for immune education and bacterial tolerance $[28,89]$. Harboring more strains of the same species is believed to be a component of a resilient microbiome as well as providing different functions [37, 90, 91]. It is likely possible to achieve microbiome health and resilience to perturbation either by increased strain-level diversity within a species or by maintenance of a diverse array of different species. In both instances, this taxonomic diversity leads to functional diversity. Intriguingly, the authors also speculated that frequent antibiotic courses could lead to multiple colonization attempts by beneficial species (e.g., Eubacterium rectale) in lieu of initial colonization and in vivo evolution. That is, because of frequent antibiotic administration, beneficial microbes are not surviving and undergoing in vivo adaptation, potentially inhibiting the microbiome plasticity expected of a healthy, interconnected, adult microbiome $[28,92]$. Consecutive samples from the same individual on antibiotics were less similar to each other than antibiotic-naive children. This result is not surprising as antibiotics are expected to acutely diminish diversity. However, these children also exhibited dramatically higher variance in this measurement even when not directly treated with antibiotics. ARGs also increased during antibiotic treatment, not all of which returned to baseline after antibiotic cessation (Table 2) [28]. Frequent antibiotic courses during childhood can thus lead to long-term consequences such as increased microbiome variability, decreased strain-level diversity, and increased MDRO potential pathogens. Thus, although the overall taxonomic structure recovers, specific entities remain in the pediatric microbiome as evidence of prior antibiotic exposure.

The main arguments against the use of antibiotics in vulnerable populations are the loss of diversity and selection for AR pathogens, which is especially worrisome when patients are immunocompromised. Treating the gut microbiota of children with severe acute malnutrition (SAM) therefore constitutes a special case combining all of the factors. In healthy children older than 2 years, some adult-like stability and resilience to antibiotics should be characteristic. Malnourished children however exhibit an age-regressed microbiome which is often lacking taxa of a healthy child or adult [39]. Specifically, the gut microbiome of children with SAM is enriched for Enterobacteriales and deficient in beneficial microbes such as Dorea spp. and Faecalibacterium prausnitzii relative to healthy children living in the same geographical area [39]. Broad-spectrum antibiotics could further deplete microbial defenses and inhibit the immune system. Indeed, the relative abundance of Enterobacteriaceae dramatically increased in children with SAM during treatment with ampicillin/amoxicillin and gentamicin [39]. The increase of potential pathogens on a background of poor microbiome and immune health could be catastrophic leading to invasive AR bacterial infections.

The data to address the long-term concerns of antimicrobial treatment of SAM is accumulating in placebocontrolled randomized trials of mass drug administration. Azithromycin and amoxicillin have been trialed to improve all-cause mortality and SAM in sub-Saharan Africa [93-95]. Researchers analyzed the gut microbiome profiles of 600 preschool children (average age 32 months) randomized to receive a standard course of oral azithromycin or placebo every 6 months for 2 years [57] (Table 2). At the end of the 2-year trial, no statistically significant differences were present between the groups in microbial richness, diversity, or phylum-level taxonomic composition. This corroborates the research above that the gross architecture is not commonly affected by a single course of antibiotics. When delving deeper into the analysis, the authors discovered a reduction in the relative abundance of 35 species including Campylobacter spp., which can cause diarrheal illnesses worldwide [57]. As a trade-off for decreased mortality, azithromycin increased phenotypic AR of pneumococcus isolated from the nares from children who completed the trial [58]. Similarly, ARGs encoding resistance to macrolides (of which azithromycin is a member) were enriched in fecal metagenomes from azithromycinexposed children. No other phenotypic resistance in pneumococcus was significantly different between the treatment groups, and there were no differences in genotypic resistance determinants in whole fecal metagenomes [57, 58] (Table 2).

Amoxicillin is also routinely prescribed for uncomplicated SAM in sub-Saharan Africa; however, similar concerns about the long-term consequences of this practice abound [96]. To address some of these concerns for amoxicillin as described above, researchers determined rates of extended-spectrum beta-lactamase (ESBL) prevalence in a randomized placebo-controlled trial of amoxicillin for SAM in Niger [93, 97]. ESBL-expressing Enterobacteriaceae broadly degrade many $\beta$-lactam antibiotics, are rising in global prevalence, are common in Asia and sub-Saharan Africa, and are listed by the CDC as serious threats $[79,98,99]$. Amoxicillin prophylaxis increased the percentage of Nigeran children who harbored ESBL Enterobacteriaceae and were negative at baseline as determined by selective culturing [97]. This result suggests either endogenous enrichment of below the limit of detection Enterobacteriaceae, de novo 
acquisition of an ESBL Enterobacteriaceae, or in vivo horizontal gene transfer (HGT). Additionally, untreated siblings of amoxicillin-treated children were more likely than placebo-controlled siblings to acquire an ESBL Enterobacteriaceae. Therefore, the administration of antibiotics can potentially affect the long-term AR of specific genera and can lead to environmental dissemination. However, given the repeated demonstration of their positive effect on childhood health, we support this practice. Further research demonstrating the environmental impact of increased AR in this population needs to be performed.

\section{Stable adulthood}

The relatively stable adult microbiome requires more sensitive measurement techniques to assess long-term perturbations. Once the microbiome has stabilized around age 2-3 [26, 35], minimal differences in microbiome stability have been observed between bi-monthly samples in children treated with various oral antibiotics [50]. Furthermore, researchers treated 66 healthy adults with one of four oral antibiotics and collected salivary and fecal samples 1, 2, 4, and 12 months after exposure [100]. Although both the fecal and salivary microbiomes were acutely disrupted by antibiotics as measured by Bray-Curtis dissimilarity, no differences persisted at 1 year suggesting long-term stability and resilience to perturbation. An alternative explanation, however, is that the microbiome is meta-stable, and antibiotic perturbation may transition the structure to an alternative, yet still stable (meta-stable) composition with similar numbers of species and diversity. Indeed, modeling existing datasets of healthy humans treated with antibiotics supports this assertion [56, 74, 100].

The response to the same antibiotic appears individualized at the taxa level likely reflective of underlying co-morbidities, local environment, or prior antibiotic history commensurate with ARG abundance (Fig. 1) [84, 101]. Additionally, the microbiome is temporally variable in relative abundance even in the absence of perturbation, but these changes were small compared to the impact of ciprofloxacin [84]. Specifically, members of the Clostridiales, among the most abundant pre-ciprofloxacin taxa, were absent in all samples after treatment [101]. Advanced sequencing techniques combined with longitudinal sampling will continue to refine our understanding of the long-term consequences and changes to the microbiome from antibiotic and environmental perturbation.

\section{Therapeutics to ameliorate microbiome and resistome disruption}

Antibiotics affect the microbiome and resistome composition, with the degree of perturbation determined by many factors (Fig. 1) [28, 55, 59]. Ultimately, if an individual's microbiome is distorted beyond correction after antibiotic cessation and treatment of an underlying condition, microbiome repair can be considered [86, 102106]. The most common diseases for which this has been trialed are Clostridioides difficile infection (CDI) and steroid non-responsive colitis [103, 105, 107]. The most common microbiota restoration methods are fecal microbiota transplant (FMT) whereby stools either from the same individual before disruption (auto) or from a healthy donor (allo) are introduced orally or via enema or probiotics. More recent research has investigated whether FMT can more generally be applied to reduce ARG and MDRO burden of resistance-rich microbiomes [108-111]. Multiple case reports have described successful decolonization of MDRO Klebsiella spp., Pseudomonas, and vancomycin-resistant enterococci with alloFMT (reviewed in [110]), though no placebo-controlled trials have yet been conducted for this express purpose. Autologous FMT has been proposed as a mechanism to restore a pre-antibiotic microbiome baseline after disruption [59, 106, 112]. Similarly, probiotics, strains of beneficial bacteria consumed during or after the intervention, are thought to mitigate some of the negative consequences of antibiotics on the microbiome [113115]. Recently, however, probiotics, autologous FMT, and spontaneous recovery were evaluated for their impact on microbiome recovery after antibiotics in healthy volunteers (Table 2) [59]. Twenty-one subjects had their stool microbiome sampled, then they were treated with 7 days of ciprofloxacin and metronidazole and randomized to twice daily probiotics for 1 month, autologous FMT from a pre-antibiotic baseline sample, or no treatment. Antibiotics disrupted gut microbiome richness and diversity and metabolic pathways devoted to sugar, carbon, and amino acid metabolism [59]. Auto-FMT immediately after antibiotics rapidly corrected this dysbiosis with no difference in Bray-Curtis dissimilarity from baseline 1 day after FMT. Spontaneous microbiome recovery occurred 21 days after antibiotic cessation. Surprisingly, probiotic administration (a commercially available 11 species consortium called Bio-25 [116]) delayed and prevented microbiome structural and functional recovery with significant compositional differences present 5 months after antibiotic cessation. The increased relative abundance of Enterococcus casseliflavus and Blautia producta correlated with maintained low species richness [59]. An important caveat to this study is that the probiotic was administered after antibiotics in lieu of during treatment which is common in clinical practice [114]. This probiotic has also not been evaluated for its prevention of antibiotic-associated diarrhea, and unless the probiotic species are resistant to the administered antibiotics, they would likely be eradicated 
during antibiotic treatment. Thus, autologous FMT accelerated and probiotic administration prevented microbiome recovery after antibiotic administration. Although probiotic administration is a common practice, these data suggest this practice may exacerbate microbiome recovery and requires further study.

Despite the benefits of FMT detailed above, the introduction of fecal microbes into another individual is not without risk. Recently, two immunocompromised patients became bacteremic with ESBL producing $E$. coli derived from donor FMT, one of whom succumbed to the infection [117]. Accordingly, the FDA has decreed that all investigational FMT products be screened specifically for MDRO, excluding individuals at higher risk for MDRO colonization and rejecting donor stools that contain MDRO [118]. One other intriguing point shown in recent FMT studies is that during the dynamic period of engraftment, new strains, species, and ARGs appear that were not detectable in either the donor or the recipient $[86,105]$. The frequency and reproducibility of this effect show that the post-treatment environment remains a major factor in the ultimate composition of the gut microbiome (Fig. 1). The sheer number of recent metagenomically sequenced FMT studies with publicly available data makes this phenomenon an excellent candidate for a meta-analysis. Accordingly, the fate of the microbiome after antibiotic perturbation depends on the environment, resilience, and availability of microbes in the post-disruption period including FMT or probiotics.

\section{Conclusions and future directions}

The state of the microbiome, the duration, route, and spectrum of antibiotic activity, other co-morbidities, diet, and post-antibiotic environment all factor into the expected acute and chronic disruption and resilience from perturbation (Fig. 1) [7, 28, 32, 43, 55, 57, 59, 74]. With the reduction of sequencing costs and technology coupled with computational pipelines, we are well poised to conduct deep and well-controlled studies of the impact of microbiome and resistome changes on human health. The most important issue for the microbiome field in general is progression from understanding correlation to identifying causal molecular mechanisms [23, $119,120]$. We are entering an era of personalized microbiome medicine, whereby medications or therapies can be tailored not only to the human genetic polymorphisms, but also to the specific microbiome constituents $[59,116]$. We can envision in the not too distant future, antimicrobials and therapies are prescribed for their direct anti-pathogen benefit while simultaneously limiting collateral damage to the microbiome and resistome [113, $121,122]$. It is naive to assume that such direct agents will not have their own collateral impacts on microbiome composition [122], but hopefully selective agents will be less disruptive overall. The human gut microbiome is intimately linked to human health and disease $[7,21,123]$. Only through carefully considering the impact of interventions on the microbiome, can we better treat diseases and improve human health.

\section{Abbreviations}

ARG: Antibiotic resistance-gene; AR: Antibiotic-resistant/resistance; NICU: Neonatal intensive care unit; IBD: Inflammatory bowel disease; ESBL: Extended spectrum beta-lactamase; SAM: Severe acute malnutrition; MDRO: Multi-drug resistant organism; FMT: Fecal microbiota transplant; HGT: Horizontal gene transfer

\section{Acknowledgements}

We would like to acknowledge the helpful comments from Alaric D'Souza and Kimberly Sukhum from the Dantas Laboratory.

\section{Authors' contributions}

DJS and AEL wrote the manuscript. GD edited the manuscript. The author(s) read and approved the final manuscript.

\section{Funding}

Individual authors were supported by the following sources: PIDS St. Jude Fellowship in Basic Research to DJS and NIH TL1 TR000449 to AL. This work was funded in part through awards to GD from the Centers for Disease Control and Prevention Epicenter Prevention Program Grant (1U1CI000033 301) and the National Center for Complementary and Integrative Health and the Eunice Kennedy Shriver National Institute of Child Health \& Human Development of the National Institutes of Health $(\mathrm{NIH})$ under award numbers R01Al123394 and R01HD092414, respectively. The content is solely the responsibility of the authors and does not necessarily represent the official views of the funding agencies.

\section{Availability of data and materials}

The data described in this review are published in the cited manuscripts.

Ethics approval and consent to participate Not applicable

Consent for publication

Not applicable

\section{Competing interests}

The authors declare that they have no competing interests.

\section{Author details}

${ }^{1}$ Department of Pediatrics, Division of Infectious Diseases, Washington University School of Medicine in St. Louis, St. Louis, MO 63110, USA. ${ }^{2}$ The Edison Family Center for Genome Sciences \& Systems Biology, Washington University School of Medicine in St. Louis, St. Louis, MO 63110, USA. ${ }^{3}$ Department of Pathology and Immunology, Division of Laboratory and Genomic Medicine, Washington University School of Medicine in St. Louis, St. Louis, MO 63110, USA. ${ }^{4}$ Department of Biomedical Engineering, Washington University in St. Louis, St. Louis, MO 63110, USA. ${ }^{5}$ Department of Molecular Microbiology, Washington University School of Medicine in St. Louis, St. Louis, MO 63110, USA.

Received: 11 March 2020 Accepted: 11 September 2020 Published online: 28 September 2020

\section{References}

1. Lynch SV, Pedersen O. The human intestinal microbiome in health and disease. N Engl J Med. 2016;375(24):2369-79.

2. Kau AL, Ahern PP, Griffin NW, Goodman AL, Gordon Jl. Human nutrition, the gut microbiome and the immune system. Nature. 2011;474(7351):327-36.

3. David LA, Maurice CF, Carmody RN, Gootenberg DB, Button JE, Wolfe BE, et al. Diet rapidly and reproducibly alters the human gut microbiome. Nature. 2014:505(7484):559-63.

4. Sharon G, Sampson TR, Geschwind DH, Mazmanian SK. The central nervous system and the gut microbiome. Cell. 2016;167(4):915-32. 
5. Stokholm J, Blaser MJ, Thorsen J, Rasmussen MA, Waage J, Vinding RK, et al. Maturation of the gut microbiome and risk of asthma in childhood. Nat Commun. 2018;9(1):141.

6. Valdes AM, Walter J, Segal E, Spector TD. Role of the gut microbiota in nutrition and health. BMJ. 2018;361:k2179.

7. Lloyd-Price J, Arze C, Ananthakrishnan AN, Schirmer M, Avila-Pacheco J, Poon TW, et al. Multi-omics of the gut microbial ecosystem in inflammatory bowel diseases. Nature. 2019;569(7758):655-62.

8. Tasnim N, Abulizi N, Pither J, Hart MM, Gibson DL. Linking the gut microbial ecosystem with the environment: does gut health depend on where we live? Front Microbiol. 2017:8:1935.

9. Stalder T, Press MO, Sullivan S, Liachko I, Top EM. Linking the resistome and plasmidome to the microbiome. ISME J. 2019;13(10):2437-46.

10. Crofts TS, Gasparrini AJ, Dantas G. Next-generation approaches to understand and combat the antibiotic resistome. Nature Reviews Microbiology. 2017;15(7):nrmicro.2017.28.

11. Truong DT, Franzosa EA, Tickle TL, Scholz M, Weingart G, Pasolli E, et al. MetaPhIAn2 for enhanced metagenomic taxonomic profiling. Nat Methods. 2015;12(10):902 -3-3.

12. Callahan BJ, McMurdie PJ, Rosen MJ, Han AW, Johnson AJA, Holmes SP. DADA2: High-resolution sample inference from Illumina amplicon data. Nature Methods. 2016;13(7):nmeth.3869.

13. Wood DE, Lu J, Langmead B. Improved metagenomic analysis with Kraken 2. Genome Biol. 2019;20(1):257.

14. Browne HP, Forster SC, Anonye BO, Kumar N, Neville BA, Stares MD, et al. Culturing of 'unculturable' human microbiota reveals novel taxa and extensive sporulation. Nature. 2016;533(7604):543-6.

15. Sommer MO, Church GM, Dantas G. The human microbiome harbors a diverse reservoir of antibiotic resistance genes. Virulence. 2010;1(4):299-303.

16. Kaminski J, Gibson MK, Franzosa EA, Segata N, Dantas G, Huttenhower C High-specificity targeted functional profiling in microbial communities with ShortBRED. PLoS Comput Biol. 2015;11(12):e1004557.

17. Franzosa EA, Mclver LJ, Rahnavard G, Thompson LR, Schirmer M, Weingart $\mathrm{G}$, et al. Species-level functional profiling of metagenomes and metatranscriptomes. Nat Methods 2018;15(11):962-8-8.

18. Gibson MK, Forsberg KJ, Dantas G. Improved annotation of antibiotic resistance determinants reveals microbial resistomes cluster by ecology. ISME J. 2015;9(1):207-16.

19. Durrant MG, Li MM, Siranosian BA, Montgomery SB, Bhatt AS. A bioinformatic analysis of integrative mobile genetic elements highlights their role in bacterial adaptation. Cell Host Microbe. 2020;27(1):140-53 e9.

20. Sberro H, Fremin BJ, Zlitni S, Edfors F, Greenfield N, Snyder MP, et al. Largescale analyses of human microbiomes reveal thousands of small. Novel Genes Cell. 2019:178(5):1245-59 e14.

21. Gilbert JA, Blaser MJ, Caporaso JG, Jansson JK, Lynch SV, Knight R. Current understanding of the human microbiome. Nat Med. 2018;24(4):392-400.

22. Knight R, Vrbanac A, Taylor BC, Aksenov A, Callewaert C, Debelius J, et al. Best practices for analysing microbiomes. Nat Rev Microbiol. 2018;16(7):410.

23. Walter J, Armet AM, Finlay BB, Shanahan F. Establishing or exaggerating causality for the gut microbiome: lessons from human microbiotaassociated rodents. Cell. 2020;180(2):221-32.

24. Gopalakrishnan V, Spencer CN, Nezi L, Reuben A, Andrews MC, Karpinets TV, et al. Gut microbiome modulates response to anti-PD-1 immunotherapy in melanoma patients. Science (New York, NY). 2018;359(6371):97-103.

25. Smith MI, Yatsunenko T, Manary MJ, Trehan I, Mkakosya R, Cheng J, et al. Gut microbiomes of Malawian twin pairs discordant for kwashiorkor. Science (New York, NY). 2013;339(6119):548-54.

26. Stewart CJ, Ajami NJ, O'Brien JL, Hutchinson DS, Smith DP, Wong MC, et al. Temporal development of the gut microbiome in early childhood from the TEDDY study. Nature. 2018;562(7728):583 -8-8.

27. Baumann-Dudenhoeffer AM, D'Souza AW, Tarr PI, Warner BB, Dantas G. Infant diet and maternal gestational weight gain predict early metabolic maturation of gut microbiomes. Nat Med. 2018;24(12):1822 -9-9.

28. Yassour M, Vatanen T, Siljander H, Hamalainen AM, Harkonen T, Ryhanen SJ, et al. Natural history of the infant gut microbiome and impact of antibiotic treatment on bacterial strain diversity and stability. Science Transl Med. 2016;8(343):343ra81.

29. Vincent C, Miller MA, Edens TJ, Mehrotra S, Dewar K, Manges AR. Bloom and bust: intestinal microbiota dynamics in response to hospital exposures and Clostridium difficile colonization or infection. Microbiome. 2016:4:12.
30. D'Souza AW, Potter RF, Wallace M, Shupe A, Patel S, Sun X, et al. Spatiotemporal dynamics of multidrug resistant bacteria on intensive care unit surfaces. Nat Commun. 2019;10(1):4569.

31. Maier L, Pruteanu M, Kuhn M, Zeller G, Telzerow A, Anderson EE, et al. Extensive impact of non-antibiotic drugs on human gut bacteria. Nature. 2018;555(7698):623-8.

32. Gasparrini AJ, Wang B, Sun X, Kennedy EA, Hernandez-Leyva A, Ndao IM, et al. Persistent metagenomic signatures of early-life hospitalization and antibiotic treatment in the infant gut microbiota and resistome. Nat Microbiol. 2019;4(12):2285-97.

33. Chu DM, Ma J, Prince AL, Antony KM, Seferovic MD, Aagaard KM. Maturation of the infant microbiome community structure and function across multiple body sites and in relation to mode of delivery. Nat Med. 2017;23(3):314-26

34. Lim ES, Zhou Y, Zhao G, Bauer IK, Droit L, Ndao IM, et al. Early life dynamics of the human gut virome and bacterial microbiome in infants. Nat Med. 2015;21(10):1228-34.

35. Yatsunenko T, Rey FE, Manary MJ, Trehan I, Dominguez-Bello MG, Contreras $M$, et al. Human gut microbiome viewed across age and geography. Nature. 2012;486(7402):222-7.

36. Yassour M, Jason E, Hogstrom LJ, Arthur TD, Tripathi S, Siljander H, et al. Strain-level analysis of mother-to-child bacterial transmission during the first few months of life. Cell Host Microbe. 2018;24(1):146-54.e4-54.e4.

37. Vatanen T, Plichta DR, Somani J, Munch PC, Arthur TD, Hall AB, et al Genomic variation and strain-specific functional adaptation in the human gut microbiome during early life. Nat Microbiol. 2019;4(3):470-9.

38. Hill CJ, Lynch DB, Murphy K, Ulaszewska M, Jeffery IB, O'Shea CA, et al. Evolution of gut microbiota composition from birth to 24 weeks in the INFANTMET Cohort. Microbiome. 2017;5(1):4

39. Subramanian S, Huq S, Yatsunenko T, Haque R, Mahfuz M, Alam MA, et al. Persistent gut microbiota immaturity in malnourished Bangladeshi children. Nature. 2014:510(7505):417-21.

40. David LA, Materna AC, Friedman J, Campos-Baptista MI, Blackburn MC, Perrotta A, et al. Host lifestyle affects human microbiota on daily timescales. Genome Biol. 2014;15(7):R89.

41. Faith JJ, Guruge JL, Charbonneau M, Subramanian S, Seedorf H, Goodman $\mathrm{AL}$, et al. The long-term stability of the human gut microbiota. Science (New York, NY). 2013;341(6141):1237439.

42. Caporaso JG, Lauber CL, Costello EK, Berg-Lyons D, Gonzalez A, Stombaugh , et al. Moving pictures of the human microbiome. Genome Biol. 2011 12(5):R50.

43. Mehta RS, Abu-Ali GS, Drew DA, Lloyd-Price J, Subramanian A, Lochhead P, et al. Stability of the human faecal microbiome in a cohort of adult men. Nat Microbiol. 2018;3(3):347 -55-55.

44. McLaren MR, Willis AD, Callahan BJ. Consistent and correctable bias in metagenomic sequencing experiments. eLife. 2019;8e46923.

45. Schmieder R, Edwards R. Fast identification and removal of sequence contamination from genomic and metagenomic datasets. PLoS One. 2011; 6(3):e17288.

46. Davis NM, Proctor DM, Holmes SP, Relman DA, Callahan BJ. Simple statistical identification and removal of contaminant sequences in marker-gene and metagenomics data. Microbiome. 2018;6(1):226.

47. Minich JJ, Sanders JG, Amir A, Humphrey G, Gilbert JA, Knight R. Quantifying and understanding well-to-well contamination in microbiome research. mSystems. 2019:4(4):e00186-19.

48. Flores GE, Caporaso JG, Henley JB, Rideout JR, Domogala D, Chase J, et al. Temporal variability is a personalized feature of the human microbiome. Genome Biol. 2014;15(12):531.

49. Costello EK, Stagaman K, Dethlefsen L, Bohannan BJ, Relman DA. The application of ecological theory toward an understanding of the human microbiome. Science (New York, NY). 2012;336(6086):1255-62.

50. Vatanen T, Franzosa EA, Schwager R, Tripathi S, Arthur TD, Vehik K, et al. The human gut microbiome in early-onset type 1 diabetes from the TEDDY Study. Nature. 2018:562(7728):589-94-94

51. D'Souza AW, Moodley-Govender E, Berla B, Kelkar T, Wang B, Sun X, et al. Cotrimoxazole prophylaxis increases resistance gene prevalence and alphadiversity but decreases beta-diversity in the gut microbiome of HIVexposed, uninfected infants. Clin Infect Dis. 2019:ciz1186.

52. Parnanen K, Karkman A, Hultman J, Lyra C, Bengtsson-Palme J, Larsson DGJ, et al. Maternal gut and breast milk microbiota affect infant gut antibiotic resistome and mobile genetic elements. Nat Commun. 2018;9(1):3891. 
53. Nogacka A, Salazar N, Suarez M, Milani C, Arboleya S, Solis G, et al. Impact of intrapartum antimicrobial prophylaxis upon the intestinal microbiota and the prevalence of antibiotic resistance genes in vaginally delivered full-term neonates. Microbiome. 2017:5(1):93.

54. Gibson MK, Wang B, Ahmadi S, Burnham C-AD, Tarr PI, Warner BB, et al. Developmental dynamics of the preterm infant gut microbiota and antibiotic resistome. Nature Microbiology. 2016;1 (4):nmicrobiol201624.

55. Bokulich NA, Chung J, Battaglia T, Henderson N, Jay M, Li H, et al. Antibiotics, birth mode, and diet shape microbiome maturation during early life. Science Translational Medicine. 2016:8(343):343ra82.

56. Palleja A, Mikkelsen KH, Forslund SK, Kashani A, Allin KH, Nielsen T, et al. Recovery of gut microbiota of healthy adults following antibiotic exposure. Nat Microbiol. 2018;3(11):1255 -65-65.

57. Doan T, Hinterwirth A, Worden L, Arzika AM, Maliki R, Abdou A, et al. Gut microbiome alteration in MORDOR I: a community-randomized trial of mass azithromycin distribution. Nat Med. 2019;25(9):1370-6.

58. Doan T, Arzika AM, Hinterwirth A, Maliki R, Zhong L, Cummings S, et al. Macrolide resistance in MORDOR I - a cluster-randomized trial in Niger. N Engl J Med. 2019;380(23):2271-3.

59. Suez J, Zmora N, Zilberman-Schapira G, Mor U, Dori-Bachash M, Bashiardes $S$, et al. Post-antibiotic gut mucosal microbiome reconstitution is impaired by probiotics and improved by autologous FMT. Cell. 2018;174(6):1406-23 e16.

60. Schulman J, Dimand RJ, Lee HC, Duenas GV, Bennett MV, Gould JB. Neonatal intensive care unit antibiotic use. Pediatrics. 2015;135(5):826-33.

61. Cantey JB, Wozniak PS, Pruszynski JE, Sanchez PJ. Reducing unnecessary antibiotic use in the neonatal intensive care unit (SCOUT): a prospective interrupted time-series study. Lancet Infect Dis. 2016;16(10):1178-84.

62. Cantey JB, Wozniak PS, Sanchez PJ. Prospective surveillance of antibiotic use in the neonatal intensive care unit: results from the SCOUT study. Pediatr Infect Dis J. 2015;34(3):267-72.

63. Schulman J, Profit J, Lee HC, Duenas G, Bennett MV, Parucha J, et al. Variations in neonatal antibiotic use. Pediatrics. 2018;142(3):e20180115.

64. Shipp KD, Chiang T, Karasick S, Quick K, Nguyen ST, Cantey JB. Antibiotic stewardship challenges in a referral neonatal intensive care unit. Am J Perinatol. 2016;33(5):518-24.

65. Dong Y, Speer CP. Late-onset neonatal sepsis: recent developments. Arch Dis Child Fetal Neonatal Ed. 2015;100(3):F257.

66. Brooks B, Firek BA, Miller CS, Sharon I, Thomas BC, Baker R, et al. Microbes in the neonatal intensive care unit resemble those found in the gut of premature infants. Microbiome. 2014;2(1):1.

67. Sommer MO, Dantas G, Church GM. Functional characterization of the antibiotic resistance reservoir in the human microflora. Science (New York, NY). 2009;325(5944):1128-31.

68. Jia B, Raphenya AR, Alcock B, Waglechner N, Guo P, Tsang KK, et al. CARD 2017: expansion and model-centric curation of the comprehensive antibiotic resistance database. Nucleic Acids Res. 2017:45(D1):D566-d73.

69. Fouhy F, Watkins C, Hill CJ, O'Shea CA, Nagle B, Dempsey EM, et al. Perinatal factors affect the gut microbiota up to four years after birth. Nat Commun. 2019;10(1):1517.

70. Stewart CJ, Skeath T, Nelson A, Fernstad SJ, Marrs EC, Perry JD, et al. Preterm gut microbiota and metabolome following discharge from intensive care. Sci Rep. 2015:5:17141.

71. Dawson-Hahn EE, Mickan S, Onakpoya I, Roberts N, Kronman M, Butler CC, et al. Short-course versus long-course oral antibiotic treatment for infections treated in outpatient settings: a review of systematic reviews. Fam Pract. 2017:34(5):511-9.

72. Lewis JD, Chen EZ, Baldassano RN, Otley AR, Griffiths AM, Lee D, et al. Inflammation, antibiotics, and diet as environmental stressors of the gut microbiome in pediatric Crohn's disease. Cell Host Microbe. 2015;18(4):489500.

73. Willmann M, Vehreschild M, Biehl LM, Vogel W, Dorfel D, Hamprecht A, et al. Distinct impact of antibiotics on the gut microbiome and resistome: a longitudinal multicenter cohort study. BMC Biol. 2019;17(1):76.

74. Shaw LP, Bassam H, Barnes CP, Walker AS, Klein N, Balloux F. Modelling microbiome recovery after antibiotics using a stability landscape framework. ISME J. 2019;13(7):1845-56

75. Raymond F, Ouameur AA, Deraspe M, labal N, Gingras H, Dridi B, et al. The initial state of the human gut microbiome determines its reshaping by antibiotics. ISME J. 2016:10(3):707-20.
76. Li J, Rettedal EA, van der Helm E, Ellabaan M, Panagiotou G, Sommer MOA. Antibiotic treatment drives the diversification of the human gut resistome. Genomics Proteomics Bioinformatics. 2019;17(1):39-51.

77. Buelow E, Bello Gonzalez TDJ, Fuentes S, de Steenhuijsen Piters WAA, Lahti $L$, Bayjanov JR, et al. Comparative gut microbiota and resistome profiling of intensive care patients receiving selective digestive tract decontamination and healthy subjects. Microbiome. 2017;5(1):88.

78. Kantele A, Laaveri T, Mero S, Vilkman K, Pakkanen SH, Ollgren J, et al. Antimicrobials increase travelers' risk of colonization by extended-spectrum betalactamase-producing Enterobacteriaceae. Clinical Infectious Diseases. 2015;60(6):837-46.

79. Arcilla MS, van Hattem JM, Haverkate MR, Bootsma MCJ, van Genderen PJJ, Goorhuis A, et al. Import and spread of extended-spectrum beta-lactamaseproducing Enterobacteriaceae by international travellers (COMBAT study): a prospective, multicentre cohort study. Lancet Infect Dis. 2017;17(1):78-85.

80. Gevers D, Kugathasan S, Denson LA, Vazquez-Baeza Y, Van Treuren W, Ren $B$, et al. The treatment-naive microbiome in new-onset Crohn's disease. Cell Host Microbe. 2014;15(3):382-92.

81. Salazar N, Valdes-Varela L, Gonzalez S, Gueimonde M, de Los Reyes-Gavilan CG. Nutrition and the gut microbiome in the elderly. Gut Microbes. 2017; 8(2):82-97.

82. Salazar N, Arboleya S, Valdes L, Stanton C, Ross P, Ruiz L, et al. The human intestinal microbiome at extreme ages of life. Dietary intervention as a way to counteract alterations. Front Genet. 2014;5:406.

83. Claesson MJ, Cusack S, O'Sullivan O, Greene-Diniz R, de Weerd H, Flannery E, et al. Composition, variability, and temporal stability of the intestinal microbiota of the elderly. Proc Natl Acad Sci U S A. 2011;108(Suppl 1):458691.

84. Dethlefsen $\mathrm{L}$, Relman DA. Incomplete recovery and individualized responses of the human distal gut microbiota to repeated antibiotic perturbation. Proc Natl Acad Sci U S A. 2011;108(Suppl 1):4554-61.

85. Isaac S, Scher JU, Djukovic A, Jimenez N, Littman DR, Abramson SB, et al. Short- and long-term effects of oral vancomycin on the human intestinal microbiota. J Antimicrob Chemother. 2017;72(1):128-36.

86. Smillie CS, Sauk J, Gevers D, Friedman J, Sung J, Youngster I, et al. Strain tracking reveals the determinants of bacterial engraftment in the human gut following fecal microbiota transplantation. Cell Host Microbe. 2018;23(2): 229-40 e5-40.e5.

87. Langdon A, Crook N, Dantas G. The effects of antibiotics on the microbiome throughout development and alternative approaches for therapeutic modulation. Genome Medicine. 2016;8(1):39.

88. Jernberg C, Lofmark S, Edlund C, Jansson JK. Long-term impacts of antibiotic exposure on the human intestinal microbiota. Microbiology (Reading, England). 2010;156(Pt 11):3216-23.

89. Round JL, Lee SM, Li J, Tran G, Jabri B, Chatila TA, et al. The Toll-like receptor 2 pathway establishes colonization by a commensal of the human microbiota. Science (New York, NY). 2011:332(6032):974-7.

90. Tett A, Huang KD, Asnicar F, Fehlner-Peach H, Pasolli E, Karcher N, et al. The Prevotella copri complex comprises four distinct clades underrepresented in westernized populations. Cell Host Microbe. 2019;26(5):666-79.e7.

91. Roodgar M, Good BH, Garud NR, Martis S, Avula M, Zhou W, et al. Longitudinal linked read sequencing reveals ecological and evolutionary responses of a human gut microbiome during antibiotic treatment. bioRxiv. 2019

92. Zhao S, Lieberman TD, Poyet M, Kauffman KM, Gibbons SM, Groussin M, et al. Adaptive evolution within gut microbiomes of healthy people. Cell Host Microbe. 2019;25(5):656-67 e8.

93. Isanaka S, Langendorf C, Berthe F, Gnegne S, Li N, Ousmane N, et al. Routine amoxicillin for uncomplicated severe acute malnutrition in children. N Engl J Med. 2016;374(5):444-53.

94. Keenan JD, Bailey RL, West SK, Arzika AM, Hart J, Weaver J, et al. Azithromycin to reduce childhood mortality in sub-Saharan Africa. N Engl J Med. 2018;378(17):1583-92.

95. Trehan I, Schechtman KB, Manary MJ. Amoxicillin for severe acute malnutrition in children. N Engl J Med. 2016;375(2):191.

96. Bogoch II, Utzinger J, Lo NC, Andrews JR. Antibacterial mass drug administration for child mortality reduction: opportunities, concerns, and possible next steps. PLoS Negl Trop Dis. 2019;13(5):e0007315.

97. Maataoui N, Langendorf C, Berthe F, Bayjanov JR, van Schaik W, Isanaka S, et al. Increased risk of acquisition and transmission of ESBL-producing 
Enterobacteriaceae in malnourished children exposed to amoxicillin. J Antimicrob Chemother. 2020;75(3):709-17.

98. CDC. Antibiotic resistance threats in the United States. Atlanta: Department of Health and Human Services; 2019.

99. Woerther P-L, Angebault C, Jacquier H, Hugede H-C, Janssens A-C, Sayadi S, et al. Massive increase, spread, and exchange of extended spectrum $\beta$ lactamase-encoding genes among intestinal Enterobacteriaceae in hospitalized children with severe acute malnutrition in Niger. Clin Infect Dis. 2011;53(7):677 -85-85.

100. Zaura E, Brandt BW, Teixeira de Mattos MJ, Buijs MJ, Caspers MP, Rashid MU, et al. Same exposure but two radically different responses to antibiotics: resilience of the salivary microbiome versus long-term microbial shifts in feces. mBio. 2015;6(6):e01693-15.

101. Dethlefsen L, Huse S, Sogin ML, Relman DA. The pervasive effects of an antibiotic on the human gut microbiota, as revealed by deep 165 rRNA sequencing. PLoS Biol. 2008;6(11):e280.

102. Brandt $L$, Aroniadis OC, Mellow M, Kanatzar A, Kelly C, Park T, et al. Longterm follow-up of colonoscopic fecal microbiota transplant for recurrent Clostridium difficile infection. Am J Gastroenterol. 2012;107(7):1079-87.

103. Orenstein R, Dubberke E, Hardi R, Ray A, Mullane K, Pardi DS, et al. Safety and durability of RBX2660 (microbiota suspension) for recurrent Clostridium difficile infection: results of the PUNCH CD Study. Clin Infectious Dis. 2016; 62(5):596-602

104. DeFilipp Z, Peled JU, Li S, Mahabamunuge J, Dagher Z, Slingerland AE, et al. Third-party fecal microbiota transplantation following allo-HCT reconstitutes microbiome diversity. Blood Adv. 2018;2(7):745-53.

105. Wang Y, Wiesnoski DH, Helmink BA, Gopalakrishnan V, Choi K, DuPont HL, et al. Fecal microbiota transplantation for refractory immune checkpoint inhibitor-associated colitis. Nat Med. 2018;24(12):1804 -8-8.

106. Taur Y, Coyte K, Schluter J, Robilotti E, Figueroa C, Gjonbalaj M, et al. Reconstitution of the gut microbiota of antibiotic-treated patients by autologous fecal microbiota transplant. Science Transl Med. 2018;10(460): eaap9489.

107. Hocquart M, Lagier JC, Cassir N, Saidani N, Eldin C, Kerbaj J, et al. Early fecal microbiota transplantation improves survival in severe Clostridium difficile infections. Clin Infectious Dis. 2018;66(5):645-50.

108. Hourigan SK, Ahn M, Gibson KM, Perez-Losada M, Felix G, Weidner M, et al. Fecal transplant in children with Clostridioides difficile gives sustained reduction in antimicrobial resistance and potential pathogen burden. Open Forum Infectious Dis. 2019;6(10):ofz379.

109. Millan B, Park H, Hotte N, Mathieu O, Burguiere P, Tompkins TA, et al. Fecal microbial transplants reduce antibiotic-resistant genes in patients with recurrent Clostridium difficile infection. Clin Infectious Dis. 2016;62(12):147986.

110. Woodworth MH, Hayden MK, Young VB, Kwon JH. The role of fecal microbiota transplantation in reducing intestinal colonization with antibiotic-resistant organisms: the current landscape and future directions. Open forum infectious diseases. 2019;6(7):ofz288.

111. Dubberke ER, Mullane KM, Gerding DN, Lee CH, Louie TJ, Guthertz $\mathrm{H}$, et al. Clearance of vancomycin-resistant Enterococcus concomitant with administration of a microbiota-based drug targeted at recurrent Clostridium difficile infection. Open forum infectious diseases. 2016;3(3):ofw133.

112. Bulow C, Langdon A, Hink T, Wallace M, Reske KA, Patel S, et al. Impact of amoxicillin-clavulanate followed by autologous fecal microbiota transplantation on fecal microbiome structure and metabolic potential. mSphere. 2018;3(6):e00588-18.

113. Schwartz DJ, Rebeck ON, Dantas G. Complex interactions between the microbiome and cancer immune therapy. Crit Rev Clin Lab Sci. 2019;56(8): 567-85.

114. Hempel S, Newberry SJ, Maher AR, Wang Z, Miles JN, Shanman R, et al. Probiotics for the prevention and treatment of antibiotic-associated diarrhea: a systematic review and meta-analysis. Jama. 2012;307(18):1959-69.

115. Kabbani TA, Pallav K, Dowd SE, Villafuerte-Galvez J, Vanga RR, Castillo NE, et al. Prospective randomized controlled study on the effects of Saccharomyces boulardii CNCM I-745 and amoxicillin-clavulanate or the combination on the gut microbiota of healthy volunteers. Gut Microbes. 2017;8(1):17-32.

116. Zmora N, Zilberman-Schapira G, Suez J, Mor U, Dori-Bachash M, Bashiardes $S$, et al. Personalized gut mucosal colonization resistance to empiric probiotics is associated with unique host and microbiome features. Cell. 2018;174(6):1388-405.e21-405.e21.
117. DeFilipp Z, Bloom PP, Torres Soto M, Mansour MK, Sater MRA, Huntley MH, et al. Drug-resistant E. coli bacteremia transmitted by fecal microbiota transplant. N Engl J Med. 2019;381(21):2043-50.

118. Administration FaD. Information pertaining to additional safety protections regarding use of fecal microbiota for transplantation - screening and testing of stool donors for multi-drug resistant organisms FDA.gov2019 [updated 06/18/2019.

119. Brito IL, Alm EJ. Tracking strains in the microbiome: insights from metagenomics and models. Front Microbiol. 2016;7:712.

120. Ridaura VK, Faith JJ, Rey FE, Cheng J, Duncan AE, Kau AL, et al. Gut microbiota from twins discordant for obesity modulate metabolism in mice. Science (New York, NY). 2013;341(6150):1241214

121. Spaulding CN, Klein RD, Ruer S, Kau AL, Schreiber HL, Cusumano ZT, et al. Selective depletion of uropathogenic E. coli from the gut by a FimH antagonist. Nature. 2017;546(7659):528.

122. Divya Ganeshan S, Hosseinidoust Z. Phage therapy with a focus on the human microbiota. Antibiotics (Basel). 2019;8(3):131.

123. Young VB. The role of the microbiome in human health and disease: an introduction for clinicians. BMJ. 2017;356:j831.

\section{Publisher's Note}

Springer Nature remains neutral with regard to jurisdictional claims in published maps and institutional affiliations. 